ITP-SB-94-22

hep-th/9406063

\title{
COMPLEX STRUCTURES, DUALITY AND WZW-MODELS IN EXTENDED SUPERSPACE
}

\author{
Ivan T. Ivanov円, Byungbae Kim², and Martin Roček \\ Institute for Theoretical Physics, \\ State University of New York at Stony Brook, \\ Stony Brook, NY 11794-3840 USA
}

\begin{abstract}
We find the complex structure on the dual of a complex target space. For $N=(2,2)$ systems, we prove that the space orthogonal to the kernel of the commutator of the left and right complex structures is always integrable, and hence the kernel is parametrized by chiral and twisted chiral superfield coordinates. We then analyze the particular case of $S U(2) \times S U(2)$, and are led to a new $N=2$ superspace formulation of the $S U(2) \times U(1)$ WZW-model.
\end{abstract}

\footnotetext{
${ }^{1}$ e-mail address: iti@insti.physics.sunysb.edu

2 e-mail address: bkim@insti.physics.sunysb.edu

${ }^{3}$ e-mail address: rocek@insti.physics.sunysb.edu
} 


\section{Introduction}

The low energy sector of a string theory is conveniently described by studying maps from a Riemann surface to a target space. Classically, this gives rise to generalized harmonic map problems, and at the quantum level, this gives a description of $d=2$ quantum field theory. Duality [1, 20 is a map between different target space descriptions of the same field theory, and has given a great deal of insight into the nonperturbative structure of the space of string backgrounds [5, 6, 7]. Here we study how duality acts on complex structures on the target space. Specifically, given a target space with an hermitian complex structure preserved by the appropriate connection [8], we find the dual complex structure that is similarly compatible with respect to the metric and connection on the dual target space.

On target spaces with torsion in $N=2$ supersymmetric theories, there are two relevant complex structures [8] (see also [9, 10]). When they commute, an $N=2$ superspace description of the model can be given in terms of a potential that is a function of constrained $N=2$ superfields. In the general case, we prove that the space orthogonal to the kernel of the commutator of the complex structures is integrable. This implies that a subsector of the theory can be described as in the fully commuting case, but for the remainder of the theory, as for the general (noncommuting) case, no $N=2$ superspace description is known. However, a special case was described locally in [9]. Here we find an application of the construction of [9] by first showing that the dual of the obvious complex structures on $S U(2) \times S U(2)$ gives rise to noncommuting complex structures on $S U(2) \times U(1)$, and then using these to construct a new $N=2$ superspace action for the super WZW-model on $S U(2) \times U(1)$.

We begin by briefly reviewing some of the results of [8, 9]. In $N=1$ superspace, additional supersymmetries (beyond the one made manifest by virtue of being in superspace) are generated by complex structures on the target space [11]. The condition that these give the usual supersymmetry algebra implies that the complex structures are integrable, and the condition that these are symmetries of the action implies that the metric is hermitian with respect to the structures, and that they are covariantly constant with

\footnotetext{
${ }^{4}$ See also [3] for a simple description of some aspects, and [4] for a discussion of global issues.
} 
respect to a connection with torsion that is given in terms of the curl of a two form on the target space [8] (see also [10] for a succinct review). The key observation is that supersymmetry determines these properties, so if we find a transformation of the target space that preserves the supersymmetry, we will automatically find corresponding complex structures. Duality is such a transformation, and thus we find dual complex structures.

Extended supersymmetries are most naturally described in extended superspace. However, it is not known how to do this in general. On general dimensional grounds, an $N=2$ superspace lagrangian is expected to be simply a potential function of some scalar superfields. To get any dynamics at all, at least some of these superfields must be constrained. For example, if the target space is Kähler, then the lagrangian is the Kähler potential, and the superfields are chiral [11]. In general, when the left and the right complex structures ( $J_{ \pm}$below) commute, then the $N=2$ superspace lagrangian is known and is described in terms of chiral and twisted chiral superfields [8]. However, generically the left and right complex structures do not commute. After considering some general aspects, we find an $N=2$ superspace lagrangian for an example with noncommuting complex structures.

\section{Complex Structures and Duality}

Given a supersymmetric nonlinear $\sigma$-model lagrangian

$$
S=-\frac{1}{2 \pi} \int D^{2}\left(\left(g_{\mu \nu}+b_{\mu \nu}\right) D_{+} X^{\mu} D_{-} X^{\nu}\right)
$$

with an isometry generated by a vector field $Y$ that obeys $L_{Y}(d b)=0$, one can choose (local) coordinates such that $Y=\partial / \partial X^{0}$ and the metric $g$ and torsion potential $b$ are independent of $X^{0}$. Then one can get a new target manifold with new metric $\tilde{g}$ and torsion potential $\tilde{b}$ by dualizing [2, 1] as follows: Gauging the symmetry with gauge fields $V_{ \pm}$(replace $D_{ \pm} X^{0}$ with $D_{ \pm} X^{0}+V_{ \pm}$and choosing $X^{0}=0$ gauge), we get the first order lagrangian

$$
\begin{array}{r}
S=-\frac{1}{2 \pi} \int D^{2}\left[e_{00} V_{+} V_{-}+e_{i 0} D_{+} X^{i} V_{-}+e_{0 i} V_{+} D_{-}\right. \\
X^{i}+e_{i j} D_{+} X^{i} D_{-} X^{j} \\
\left.+\tilde{\phi}\left(D_{+} V_{-}+D_{-} V_{+}\right)\right]
\end{array}
$$

Here $\{\mu, \nu\}=0,1, . ., 2 n-1,\{i, j\}=1, . ., 2 n-1, e_{\mu \nu}=g_{\mu \nu}+b_{\mu \nu}$, and $\tilde{\phi}$ is the lagrange multiplier whose variation imposes $V_{ \pm}=D_{ \pm} X^{0}$ and gives back the 
original action (11). Extended $(2,2)$ supersymmetry of the action (11) implies that there is an invariance $\delta_{\varepsilon} X^{\mu}=\varepsilon J_{+\nu}^{\mu} D_{+} X^{\nu}$ and $\delta_{\eta} X^{\mu}=\eta J_{-\nu}^{\mu} D_{-} X^{\nu}$ where $J_{ \pm}$are integrable complex structures that are covariantly constant with respect to a connection with torsion $T_{\mu \nu \rho}= \pm \frac{1}{2}\left(\partial_{\mu} b_{\nu \rho}+\partial_{\nu} b_{\rho \mu}+\partial_{\rho} b_{\mu \nu}\right)$ [8, 3, 10]. The first order action (2) is invariant under

$$
\begin{gathered}
\delta_{\varepsilon} \tilde{\phi}=e_{\mu 0} \varepsilon\left(J_{+0}^{\mu} V_{+}+J_{+j}^{\mu} D_{+} X^{j}\right), \quad \delta_{\varepsilon} X^{i}=\varepsilon\left(J_{+0}^{i} V_{+}+J_{+j}^{i} D_{+} X^{j}\right) \\
\delta_{\varepsilon} V_{+}=D_{+} \delta_{\varepsilon} X^{0} \equiv D_{+}\left[\varepsilon\left(J_{+0}^{0} V_{+}+J_{+i}^{0} D_{+} X^{i}\right)\right]
\end{gathered}
$$

and

$$
\begin{gathered}
\delta_{\eta} \tilde{\phi}=-e_{0 \mu} \eta\left(J_{-0}^{\mu} V_{-}+J_{-j}^{\mu} D_{-} X^{j}\right), \quad \delta_{\eta} X^{i}=\eta\left(J_{-0}^{i} V_{-}+J_{-j}^{i} D_{-} X^{j}\right), \\
\delta_{\eta} V_{-}=D_{-} \delta_{\eta} X^{0} \equiv D_{-}\left[\eta\left(J_{-0}^{0} V_{-}+J_{-i}^{0} D_{-} X^{i}\right)\right] .
\end{gathered}
$$

To find the dual model, we eliminate $V_{ \pm}$by their equations of motion

$$
V_{+}=e_{00}^{-1}\left(D_{+} \tilde{\phi}-e_{i 0} D_{+} X^{i}\right), \quad V_{-}=-e_{00}^{-1}\left(D_{-} \tilde{\phi}+e_{0 i} D_{-} X^{i}\right)
$$

so that

$$
\begin{aligned}
\delta_{\varepsilon} \tilde{\phi} & =\varepsilon\left[e_{\mu 0} J_{+0}^{\mu} e_{00}^{-1} D_{+} \tilde{\phi}+\left(-e_{\mu 0} J_{+0}^{\mu} e_{00}^{-1} e_{i 0}+e_{\mu 0} J_{+i}^{\mu}\right) D_{+} X^{i}\right], \\
\delta_{\varepsilon} X^{j} & =\varepsilon\left[J_{+0}^{j} e_{00}^{-1} D_{+} \tilde{\phi}+\left(J_{+i}^{j}-J_{+0}^{j} e_{00}^{-1} e_{i 0}\right) D_{+} X^{i}\right], \\
\delta_{\eta} \tilde{\phi} & =\eta\left[e_{0 \mu} J_{-0}^{\mu} e_{00}^{-1} D_{-} \tilde{\phi}+\left(e_{0 \mu} J_{-0}^{\mu} e_{00}^{-1} e_{0 i}-e_{0 \mu} J_{-i}^{\mu}\right) D_{-} X^{i}\right], \\
\delta_{\eta} X^{j} & =-\eta\left[J_{-0}^{j} e_{00}^{-1} D_{-} \tilde{\phi}-\left(J_{-i}^{j}-J_{-0}^{j} e_{00}^{-1} e_{0 i}\right) D_{-} X^{i}\right] .
\end{aligned}
$$

From the transformations (8-[1), we can read off the dual complex structures $K_{+}$and $K_{-}$:

$$
K_{+\nu}^{\mu}=\left(\begin{array}{cc}
e_{\mu 0} J_{+0}^{\mu} e_{00}^{-1} & -e_{\mu 0} J_{+0}^{\mu} e_{00}^{-1} e_{i 0}+e_{\mu 0} J_{+i}^{\mu} \\
J_{+0}^{j} e_{00}^{-1} & J_{+i}^{j}-J_{+0}^{j} e_{00}^{-1} e_{i 0}
\end{array}\right)
$$


and

$$
K_{-\nu}^{\mu}=\left(\begin{array}{cc}
e_{0 \mu} J_{-0}^{\mu} e_{00}^{-1} & e_{0 \mu} J_{-0}^{\mu} e_{00}^{-1} e_{0 i}-e_{0 \mu} J_{-i}^{\mu} \\
-J_{-0}^{j} e_{00}^{-1} & J_{-i}^{j}-J_{-0}^{j} e_{00}^{-1} e_{0 i}
\end{array}\right)
$$

with respect to $\left\{d \tilde{\phi}, d X^{1} . . d X^{2 n-1}\right\}$.

Substituting (7) into (2), we get the new action [2]

$$
\begin{array}{r}
\tilde{S}=-\frac{1}{2 \pi} \int D^{2}\left[\frac{1}{e_{00}}\left(D_{+} \tilde{\phi} D_{-} \tilde{\phi}+e_{0 j} D_{+} \tilde{\phi} D_{-} X^{j}-e_{i 0} D_{+} X^{i} D_{-} \tilde{\phi}\right)\right. \\
\left.+\left(e_{i j}-\frac{e_{i 0} e_{0 j}}{e_{00}}\right) D_{+} X^{i} D_{-} X^{j}\right]
\end{array}
$$

and read off the dual metric $\tilde{g}$ and torsion potential $\tilde{b}$ :

$$
\tilde{g}_{\mu \nu}=\left(\begin{array}{cc}
g_{00}^{-1} & g_{00}^{-1} b_{0 i} \\
g_{00}^{-1} b_{0 i} & g_{i j}-g_{00}^{-1}\left(g_{i 0} g_{j 0}+b_{i 0} b_{0 j}\right)
\end{array}\right),
$$

and

$$
\tilde{b}_{\mu \nu}=\left(\begin{array}{cc}
0 & g_{00}^{-1} g_{0 i} \\
-g_{00}^{-1} g_{0 i} & b_{i j}+g_{00}^{-1}\left(g_{i 0} b_{j 0}-g_{j 0} b_{i 0}\right)
\end{array}\right)
$$

with respect to the same basis as above.

We end this section with some observations. If $J_{+}=J_{-}$(the Kähler case), then generally $K_{+} \neq K_{-}$, but $\left[K_{+}, K_{-}\right]=0$. Further, if we begin with $\left[J_{+}, J_{-}\right]=0$ (and $J_{+} \neq J_{-}$), then in general $\left[K_{+}, K_{-}\right] \neq 0$. In the most general case, when $\left[J_{+}, J_{-}\right] \neq 0$, the conditions for $\left[K_{+}, K_{-}\right]=0$ are:

$$
\begin{gathered}
\left\{J_{+}, J_{-}\right\}_{0}^{i}=\left[J_{+}, J_{-}\right]^{\mu}{ }_{0} g_{\mu 0}=\left\{J_{+}, J_{-}\right\}^{\mu}{ }_{i} g_{\mu 0}-\left\{J_{+}, J_{-}\right\}^{\mu}{ }_{0} g_{\mu i}=0, \\
2\left(J_{+0}^{i} J_{-0 j}-J_{-0}^{i} J_{+0 j}\right)=g_{00}\left[J_{+}, J_{-}\right]^{i}{ }_{j}-\left[J_{+}, J_{-}\right]^{i}{ }_{0} g_{0 j},
\end{gathered}
$$

where $J_{0 j} \equiv J^{\mu}{ }_{j} g_{\mu 0}$. 


\section{Integrability}

As we have seen, the right and left complex structures $J_{ \pm}$on the target manifold do not commute for generic $\sigma$-models with torsion. A simple algebraic argument shows that the kernel of the commutator $\left[J_{+}, J_{-}\right]$is the direct sum of the kernels of the sum and difference of the two complex structures:

$$
\operatorname{ker}\left[J_{+}, J_{-}\right]=\operatorname{ker}\left(J_{+}+J_{-}\right) \oplus \operatorname{ker}\left(J_{+}-J_{-}\right) \text {. }
$$

The whole manifold cannot be described in terms of chiral and twisted chiral superfields. However, if there are one-forms annihilated by $\left[J_{+}, J_{-}\right]$, and if these one-forms form an integrable Pfaff system (equivalently if $\left(\operatorname{ker}\left[J_{+}, J_{-}\right]\right)^{\perp}$ is integrable), they can be integrated to coordinates on the manifold. Here we show that the set of one-forms in the kernel of $\left[J_{+}, J_{-}\right]$is always integrable (we have also shown that the one-forms in each $\operatorname{ker}\left(J_{+} \pm J_{-}\right)$are separately integrable). These preferred coordinates are described by chiral or twisted chiral superfields, and are perpendicular to a foliation of the manifold by submanifolds on which the commutator has no kernel.p

To prove that the forms in the kernel of the commutator $\left[J_{+}, J_{-}\right]$are integrable, it is convenient to choose complex coordinates in which $J_{+}$is diagonal and constant. The vector index $\mu$ splits into holomorphic and antiholomorphic indexes $\mu \rightarrow m, \bar{m}$. In these coordinates, $J_{+}$is given by

$$
J_{+\bar{n}}^{m}=J_{+n}^{\bar{m}}=0, \quad J_{+n}^{m}=i \delta_{n}^{m}, \quad J_{+\bar{n}}^{\bar{m}}=-i \delta_{\bar{n}}^{\bar{m}},
$$

and the components of the commutator are

$$
\begin{gathered}
{\left[J_{+}, J_{-}\right]_{n}^{m}=\left[J_{+}, J_{-}\right]_{\bar{n}}^{\bar{m}}=0,} \\
{\left[J_{+}, J_{-}\right]_{\bar{n}}^{m}=2 i J_{-\bar{n}}^{m},\left[J_{+}, J_{-}\right]_{n}^{\bar{m}}=-2 i J_{-n}^{\bar{m}} .}
\end{gathered}
$$

One-forms $a_{\mu} d x^{\mu}$ in the kernel of the commutator satisfy

$$
a_{\mu}\left[J_{+}, J_{-}\right]_{\nu}^{\mu} d x^{\nu}=0,
$$

\footnotetext{
${ }^{5}$ In a previous version of this paper, we concluded that the kernel was not integrable. This was because we looked at the integrability of of the vector fields annihilated by $\left[J_{+}, J_{-}\right]$; this kernel is indeed not integrable, which implies that there does not exist a foliation by submanifolds on which $\left[J_{+}, J_{-}\right]=0$. However, such a foliation is not needed to guarantee the existence of chiral and twisted chiral superfields.
} 
which in the holomorphic coordinate system is equivalent to

$$
a_{m} J_{-\bar{n}}^{m}=a_{\bar{m}} J_{-n}^{\bar{m}}=0 \text {. }
$$

The underlying supersymmetric $\sigma$-model induces several restrictions on the complex structures; as we use a holomorphic coordinate system, only equations involving $J_{-}$are relevant. $J_{-}$is covariantly constant

$$
D_{\mu} J_{-\nu}^{\rho} \equiv \partial_{\mu} J_{-\nu}^{\rho}+\Gamma_{\lambda \mu}^{(-) \rho} J_{-\nu}^{\lambda}-\Gamma_{\nu \mu}^{(-) \lambda} J_{-\lambda}^{\rho}=0
$$

with respect to a connection with torsion

$$
\Gamma_{\mu \nu}^{(-) \lambda}=\left\{\begin{array}{l}
\lambda \\
\mu \nu
\end{array}\right\}-\frac{1}{2} T_{\mu \nu}^{\lambda},
$$

where $\left\{\begin{array}{l}\lambda \\ \mu \nu\end{array}\right\}$ is the Riemannian connection of the metric on the target manifold, and $T_{\mu \nu}^{\lambda}$ is the torsion tensor. An algebraic identity relates the torsion and the complex structure [B],

$$
T_{\mu \nu}^{\lambda}=J_{-\mu}^{\rho} J_{-\nu}^{\sigma} T_{\rho \sigma}^{\lambda}+J_{-[\nu \mid}^{\rho} J_{-\sigma}^{\lambda} T_{\rho \mid \mu]}^{\sigma} .
$$

Finally, because the metric is hermitian with respect to both complex structures, in the holomorphic coordinate system for $J_{+}$some of the components of the connection $\Gamma^{(-)}$and the torsion $T$ vanish:

$$
\begin{gathered}
\Gamma_{\bar{j} \bar{k}}^{(-) i}=\Gamma_{j k}^{(-) \bar{i}}=\Gamma_{j \bar{k}}^{(-) i}=\Gamma_{\bar{j} k}^{(-) \bar{i}}=0, \\
T_{\bar{j} \bar{k}}^{i}=T_{j k}^{\bar{i}}=0 .
\end{gathered}
$$

Let $\omega^{p}=a_{\mu}^{p} d x^{\mu}, \quad p=1, \ldots, N$ span the set one-forms in the kernel of the commutator of the two complex structures. These one-forms can be integrated simultaneously to $N$ coordinates on the manifold $\mathcal{M}$ if and only if the Frobenius integrability condition is satisfied, that is, if the algebra of the $\operatorname{dim}(\mathcal{M})-N$ vector fields $X_{q}=X_{q}^{\nu} \frac{\partial}{\partial x^{\nu}}$ orthogonal to the forms $\omega^{p}$ closes. Explicitly, for all vector fields $X$ satisfying $\omega^{p}(X) \equiv a_{\mu}^{p} X^{\mu}=0$, we must have $\left[X_{q}, X_{r}\right]=C_{q r}^{s} X_{s}$.

Here the orthogonal vector fields $X$ are (see 23):

$$
X_{\nu}=\left[J_{+}, J_{-}\right]_{\nu}^{\mu} \frac{\partial}{\partial x^{\mu}}
$$


Note that the index $\nu$ on $X_{\nu}$ runs from $1, \ldots, \operatorname{dim}(\mathcal{M})$, but only $\operatorname{dim}(\mathcal{M})-N$ vector fields $X_{\nu}$ are linearly independent. We have integrability if all the $\omega^{p}$ are annihilated by $\left[X_{\mu}, X_{\nu}\right]$, i.e., if

$$
a_{\mu}^{p}\left[J_{+}, J_{-}\right]_{\nu}^{\mu} \Rightarrow a_{\mu}^{p}\left[J_{+}, J_{-}\right]_{[\kappa}^{\nu}\left[J_{+}, J_{-}\right]_{\lambda], \nu}^{\mu}=0 .
$$

Consider first $\kappa, \lambda=k, l$ both holomorphic. Then (21) restricts the summation over $\mu$ to the antiholomorphic part: $\mu \rightarrow \bar{m}$. The integrability condition (31) becomes

$$
a_{\bar{m}} J_{-[k}^{\nu} J_{-l], \nu}^{\bar{m}}=0
$$

We eliminate the derivatives on $J_{-}$because it is covariantly constant (25)

$$
a_{\bar{m}} J_{-[k}^{\nu} \Gamma_{l] \nu}^{(-) \rho} J_{-\rho}^{\bar{m}}-a_{\bar{m}} J_{-[k}^{\nu} J_{-l]}^{\rho} \Gamma_{\rho \nu}^{(-) \bar{m}}=0 .
$$

In the first term, (24) implies that the summation over $\rho$ is only over the antiholomorphic part of that index. Because of this and (28) we can antisymmetrize the $l$ and $\nu$ subscripts of $\Gamma^{(-)}$in the first term. This picks the antisymmetric part of the connection and we obtain:

$$
a_{\bar{m}} J_{-[k}^{\nu} T_{l] \nu}^{\rho} J_{-\rho}^{\bar{m}}-a_{\bar{m}} J_{-k}^{\nu} J_{-l}^{\rho} T_{\rho \nu}^{\bar{m}}=0 .
$$

But using the identity (27) this is equivalent to

$$
a_{\bar{m}} T_{k l}^{\bar{m}}=0
$$

which is satisfied because of (29). The proof for $\lambda, \kappa$ in (31) both antiholomorphic is equivalent, and we are left to consider, e.g., $\kappa=\bar{k}$ antiholomorphic and $\lambda=l$ holomorphic. The integrability condition (31) becomes

$$
a_{\bar{m}} J_{-\bar{k}}^{n} J_{-n l, n}^{\bar{m}}-a_{m} J_{-l}^{\bar{n}} J_{-\bar{k}, \bar{n}}^{m}=0 \text {. }
$$

Each term in (36) vanishes by itself. The first one is

$$
a_{\bar{m}} J_{-\bar{k}}^{n}\left(\Gamma_{l n}^{(-) \rho} J_{-\rho}^{\bar{m}}-\Gamma_{\rho n}^{(-) \bar{m}} J_{-l}^{\rho}\right)=0
$$

The summation over $\rho$ in the first term of (37) is restricted to the antiholomorphic part because of (24) and then the whole expression (37) vanishes because the components of the connection entering it are zero (28). The second term in (36) vanishes similarly. 
Thus we have proven that the set of one-forms annihilated by the commutator of the two complex structures can always be integrated to coordinates $x^{\mu}$ on the manifold. They correspond to chiral and twisted chiral superfields. The levels $x^{\mu}=$ constant of these distinguished coordinates folliate the target manifold with submanifolds on which the commutator of the two complex structures is nondegenerate.

Actually, to conclude that the coordinates correspond to chiral and twisted chiral multiplets, we need the integrability not just of $\left(k e r\left[J_{+}, J_{-}\right]\right)^{\perp}$, but of $\left(k e r\left(J_{+} \pm J_{-}\right)\right)^{\perp}$ each separately; this can be proven using the same techniques as above. Then chiral superfields correspond to $\operatorname{ker}\left(J_{+}-J_{-}\right)$and twisted chiral superfields to $\operatorname{ker}\left(J_{+}+J_{-}\right)$.

\section{An example}

The $S U(2) \times S U(2)$ WZW model has the action $S=S_{1}+S_{2}$, where

$$
S_{1}(h)=\frac{1}{2 \pi} \int_{\partial M} \operatorname{tr}\left(h^{-1} \partial h h^{-1} \bar{\partial} h\right)+\frac{1}{2 \pi} \int_{M} \operatorname{tr}\left(h^{-1} d h \wedge h^{-1} d h \wedge h^{-1} d h\right) .
$$

and similarly for $S_{2}$. Writing $h=\exp ^{\frac{i}{2} \theta_{L} \sigma_{3}} \exp ^{\frac{i}{2} \phi \sigma_{2}} \exp ^{\frac{i}{2} \theta_{R} \sigma_{3}}$ where $h \in S U(2)$ and $\sigma_{1}, \sigma_{2}, \sigma_{3}$ are Pauli matrices, we have

$$
S_{1}(h)=\frac{1}{2 \pi} \int_{\partial M}\left(\partial \phi \bar{\partial} \phi+\partial \theta_{L} \bar{\partial} \theta_{L}+\partial \theta_{R} \bar{\partial} \theta_{R}+2 \cos \phi \partial \theta_{L} \bar{\partial} \theta_{R}\right) .
$$

The metric and the torsion potential can be read off from $S(h)$. We construct complex structures on $S U(2) \times S U(2)$ starting from the action on the Lie algebra $s u(2) \oplus s u(2)$; for example, we may choose

$$
\begin{aligned}
& I_{+}: \quad E_{ \pm}^{1,2} \rightarrow \pm i E_{ \pm}^{1,2}, \quad E_{3}^{1} \rightarrow E_{3}^{2}, \quad E_{3}^{2} \rightarrow-E_{3}^{1} \\
& I_{-}: \quad E_{ \pm}^{1,2} \rightarrow \pm i E_{ \pm}^{1,2}, \quad E_{3}^{1} \rightarrow-E_{3}^{2}, \quad E_{3}^{2} \rightarrow E_{3}^{1}
\end{aligned}
$$

where $I_{ \pm}$are expressed in a basis of left(right)-invariant frames, $e_{(L)}^{a}, e_{(R)}^{a}$ defined by $g^{-1} d g=e_{(L)}^{a} T_{a}, d g g^{-1}=e_{(R)}^{a} T_{a}$, respectively. Here $T_{1}, T_{2}, T_{3}$ are $i / 2$ times Pauli matrices and $E_{ \pm}^{1,2}=\frac{1}{\sqrt{2}}\left(T_{1} \pm i T_{2}\right), E_{3}^{1,2}=T_{3}$. Thus we find the noncommuting complex structures

$$
J_{+\nu}^{\mu}=e_{(L) a}^{\mu} I_{+b}^{a} e_{(L) \nu}^{b}, \quad J_{-\nu}^{\mu}=e_{(R) a}^{\mu} I_{-b}^{a} e_{(R) \nu}^{b} .
$$


The commutator of the complex structures $J_{ \pm}$has a two dimensional kernel. We now find the corresponding complex coordinate explicitly. In a basis of one forms arranged as $\left(d \theta_{L}^{1}, d \theta_{R}^{1}, d \phi^{1}, d \theta_{L}^{2}, d \theta_{R}^{2}, d \phi^{2}\right)$, the two complex structures are given by the following matrices:

$$
\begin{aligned}
& J_{+}=\left[\begin{array}{cccccc}
0 & 0 & -1 / \sin \phi^{1} & 0 & 0 & 0 \\
0 & 0 & \cot \phi^{1} & \cos \phi^{2} & 1 & 0 \\
\sin \phi^{1} & 0 & 0 & 0 & 0 & 0 \\
0 & 0 & 0 & 0 & 0 & -1 / \sin \phi^{2} \\
-\cos \phi^{1} & -1 & 0 & 0 & 0 & \cot \phi^{2} \\
0 & 0 & 0 & \sin \phi^{2} & 0 & 0
\end{array}\right], \\
& J_{-}=\left[\begin{array}{cccccc}
0 & 0 & -\cot \phi^{1} & -1 & -\cos \phi^{2} & 0 \\
0 & 0 & 1 / \sin \phi^{1} & 0 & 0 & 0 \\
0 & -\sin \phi^{1} & 0 & 0 & 0 & 0 \\
1 & \cos \phi^{1} & 0 & 0 & 0 & -\cot \phi^{2} \\
0 & 0 & 0 & 0 & 0 & 1 / \sin \phi^{2} \\
0 & 0 & 0 & 0 & -\sin \phi^{2} & 0
\end{array}\right] .
\end{aligned}
$$

Explicit calculation shows that $J_{+}-J_{-}$has no kernel but $J_{+}+J_{-}$vanishes on a subspace of dimension two. The two eigenforms are:

$$
\begin{aligned}
& \frac{1}{\sin \phi^{1}}\left(\cos \phi^{1}-1\right) d \phi^{1}+d \theta_{L}^{2}+d \theta_{R}^{2}, \\
& \frac{1}{\sin \phi^{2}}\left(1-\cos \phi^{2}\right) d \phi^{2}+d \theta_{L}^{1}+d \theta_{R}^{1} .
\end{aligned}
$$

These can be integrated to a complex coordinate $\eta$

$$
\eta=2 \ln \left(\cos \left(\phi^{1} / 2\right)\right)-\theta_{L}^{2}-\theta_{R}^{2}-2 i \ln \left(\cos \left(\phi^{2} / 2\right)\right)-i \theta_{L}^{1}-i \theta_{R}^{1}
$$

that is holomorphic with respect to $J_{+}$and antiholomorphic with respect $J_{-}$:

$$
d \eta J_{ \pm}= \pm i d \eta .
$$

In superspace, this coordinate is described by a twisted chiral superfield.

We now perform a duality transformation with respect to a coordinate $X^{0}=\left(\theta_{R}^{1}+\theta_{L}^{1}\right) / \sqrt{2}$ as described above in the general case, and get a new 
metric, torsion, and complex structures $K_{+}$and $K_{-}$. The new space we get is $(S U(2) / U(1)) \times(S U(2) \times U(1))$. The two complex structures $K_{+}$ and $K_{-}$commute on an integrable 2 dimensional subspace of the tangent bundle (the tangent space of the $S U(2) / U(1)$ factor), but do not commute on $S U(2) \times U(1)$; the anticommutator of $K_{+}$and $K_{-}$on $S U(2) \times U(1)$ gives $-2 I \cos \phi$, where $I$ is the identity. Explicitly, on $S U(2) / U(1)$, in a basis $\left(d \phi^{1}, d \psi \equiv \frac{d \theta_{R}^{1}-d \theta_{L}^{1}}{2}-\frac{d \tilde{\phi}}{\sqrt{2}}\right)$, where $\tilde{\phi}$ is the new dual coordinate (14), we have:

$$
K_{+}=K_{-}=\left[\begin{array}{cc}
0 & -\tan \frac{\phi^{1}}{2} \\
\cot \frac{\phi^{1}}{2} & 0
\end{array}\right] \text {. }
$$

On $U(1) \times S U(2)$, in a basis $\left(d \chi, d \theta_{L}^{2}, d \theta_{R}^{2}, \phi^{2}\right)$, where $\chi \equiv \frac{\theta_{R}^{1}-\theta_{L}^{1}}{2}+\frac{\tilde{\phi}}{\sqrt{2}}$, we have:

$$
\begin{aligned}
K_{+} & =\left[\begin{array}{cccc}
0 & \cos \phi^{2} & 1 & 0 \\
0 & 0 & 0 & -1 / \sin \phi^{2} \\
-1 & 0 & 0 & \cot \phi^{2} \\
0 & \sin \phi^{2} & 0 & 0
\end{array}\right], \\
K_{-}= & {\left[\begin{array}{cccc}
0 & 1 & \cos \phi^{2} & 0 \\
-1 & 0 & 0 & -\cot \phi^{2} \\
0 & 0 & 0 & 1 / \sin \phi^{2} \\
0 & 0 & -\sin \phi^{2} & 0
\end{array}\right] . }
\end{aligned}
$$

On $S U(2) / U(1)$ there is no torsion and the metric is

$$
\tilde{g}=\left[\begin{array}{cc}
1 & 0 \\
0 & \tan ^{2} \frac{\phi^{1}}{2}
\end{array}\right],
$$

and on $U(1) \times S U(2)$ the metric and torsion potential are

$$
\begin{gathered}
\tilde{g}=\left[\begin{array}{cccc}
1 & 0 & 0 & 0 \\
0 & 1 & \cos \phi^{2} & 0 \\
0 & \cos \phi^{2} & 1 & 0 \\
0 & 0 & 0 & 1
\end{array}\right], \\
\tilde{b}=\left[\begin{array}{cccc}
0 & 0 & 0 & 0 \\
0 & 0 & \cos \phi^{2} & 0 \\
0 & -\cos \phi^{2} & 0 & 0 \\
0 & 0 & 0 & 0
\end{array}\right] .
\end{gathered}
$$


The essential point is that though $S U(2) \times U(1)$ admits commuting left and right complex structures, duality has given us some others. This can be most easily understood if we paramatrize the group $S U(2) \times U(1)$ as (see, for example, [13]):

$$
g=\frac{e^{i \theta}}{\sqrt{\Phi \bar{\Phi}+\Lambda \bar{\Lambda}}}\left(\begin{array}{cc}
\Lambda & \bar{\Phi} \\
-\Phi & \bar{\Lambda}
\end{array}\right), \quad \theta=-\frac{1}{2} \ln (\Phi \bar{\Phi}+\Lambda \bar{\Lambda}) .
$$

Letting the imaginary quaternions $\left(\begin{array}{cc}0 & i \\ i & 0\end{array}\right),\left(\begin{array}{cc}0 & -1 \\ 1 & 0\end{array}\right),\left(\begin{array}{cc}i & 0 \\ 0 & -i\end{array}\right)$ act on $\left(\begin{array}{cc}d \Lambda & d \bar{\Phi} \\ -d \Phi & d \bar{\Lambda}\end{array}\right)$ from left or right gives two commuting sets of complex structures $J_{-}^{1}, J_{-}^{2}, J_{-}^{3}$ and $J_{+}^{1}, J_{+}^{2}, J_{+}^{3}$; in terms of these, $K_{+}$and $K_{-}$(50,51) are expressed as

$$
K_{+}=J_{+}^{3}, \quad K_{-}=\cos \phi^{2} J_{+}^{3}+\sin \phi^{2} \cos \theta_{R}^{2} J_{+}^{1}-\sin \phi^{2} \sin \theta_{R}^{2} J_{+}^{2}
$$

and it is clear their anticommutator is $-2 \cos \phi^{2}$. We can interpret this as follows: by construction, $\left[J_{+}, J_{-}\right]=0$. However, $S U(2) \times U(1)$ has an orientation reversing outer automorphism $\Omega$ that acts by taking the $U(1)$ generator $Q \rightarrow-Q$. This preserves $g$ and $b$, but not $J$; so $K_{-}=\Omega\left(J_{+}^{3}\right)$.

We summarize the situation as follows: On $S U(2) \times S U(2)$, there is no choice of $J_{+}$and $J_{-}$such that $\left[J_{+}, J_{-}\right]=0$. The dual of $S U(2) \times S U(2)$ is $S U(2) \times U(1) \times S U(2) / U(1)$; this does admit $J_{ \pm}$that commute, but the complex structures that we find by duality ( $K_{ \pm}$above) do not commute.

\section{A new $N=2$ description of $S U(2) \times U(1)$}

The essential ingredient for $N=2$ superspace in two dimensions (with Lorentzian signature) is a pair of complex spinor derivatives $D_{ \pm}$that obey the algebra (see, for example, [8, 10]):

$$
D_{ \pm}^{2}=\bar{D}_{ \pm}^{2}=\left\{D_{ \pm}, \bar{D}_{\mp}\right\}=0, \quad\left\{D_{ \pm}, \bar{D}_{ \pm}\right\}= \pm i \partial_{ \pm},
$$

where $\partial_{ \pm}$are the usual two-dimensional derivatives along the two lightlike directions. An $N=2$ superspace action is written as

$$
S=\frac{1}{2 \pi} \int D^{2} \bar{D}^{2} K\left(\Phi^{i}\right)
$$


where $K$ is some potential function of various superfields $\Phi^{i}$. As discussed in the introduction, a $(2,2)$ superfield description of arbitrary $N=2$ supersymmetric sigma-models (in particular, WZW-models) is not known unless $J_{ \pm}$commute. In that case, the model can be described in terms of chiral superfields $\Phi\left(\bar{D}_{ \pm} \Phi=0\right)$ and twisted chiral superfields $\Lambda\left(\bar{D}_{+} \Lambda=D_{-} \Lambda=0\right)$ [9]. The simplest example is $S U(2) \times U(1)$, where we parametrize the group element as 13

$$
g=\frac{e^{i \theta}}{\sqrt{\Phi \bar{\Phi}+\Lambda \bar{\Lambda}}}\left(\begin{array}{cc}
\Lambda & \bar{\Phi} \\
-\Phi & \bar{\Lambda}
\end{array}\right), \quad \theta=-\frac{1}{2} \ln (\Phi \bar{\Phi}+\Lambda \bar{\Lambda})
$$

and the superspace Lagrangian is

$$
K=-\int^{\frac{\Lambda \bar{\Lambda}}{\Phi \Phi}} \frac{d \zeta}{\zeta} \ln (1+\zeta)+\frac{1}{2}(\ln (\Phi \bar{\Phi}))^{2} .
$$

By construction, the manifest complex structures are the commuting ones. We now give an $N=2$ superspace description with respect to the noncommuting complex structures $K_{ \pm}$we found above.

To do this, we use a different superspace description in terms of semichiral and anti-semichiral superfields $\Phi_{1}, \bar{\Phi}_{1}, \Phi_{2}, \bar{\Phi}_{2}$ (which obey $\bar{D}_{+} \Phi_{1}=\bar{D}_{-} \Phi_{2}=$ $D_{+} \bar{\Phi}_{1}=D_{-} \bar{\Phi}_{2}=0$ ) [9]. We find the action by a new kind of duality transformation. Then by construction we have the same metric and torsion, but different complex structures. More explicitly, we add lagrange multiplier terms to the lagrangian (60) to get the first order lagrangian, [

$$
\begin{gathered}
K=-\int^{x} \frac{d \zeta}{\zeta} \ln (1+\zeta)+\frac{1}{2}(\ln y)^{2}+\left(\Phi_{1}+\Phi_{2}\right) e^{i \theta} y+\left(\bar{\Phi}_{1}+\bar{\Phi}_{2}\right) e^{-i \theta} y \\
+\left(\Phi_{1}+\bar{\Phi}_{2}\right) x y e^{i \phi}+\left(\bar{\Phi}_{1}+\Phi_{2}\right) x y e^{-i \phi}
\end{gathered}
$$

where

$$
x=\frac{\Lambda \bar{\Lambda}}{\Phi \bar{\Phi}}, \quad y=\Phi \bar{\Phi}, \quad e^{i \theta}=\frac{\Phi}{\bar{\Phi}}, \quad e^{i \phi}=\frac{\Lambda}{\bar{\Lambda}}
$$

(here $\Phi, \bar{\Phi}, \Lambda, \bar{\Lambda}$, and hence $x, y, \theta, \phi$, are now unconstrained superfields).

\footnotetext{
${ }^{6}$ This duality superficially resembles a well-known and trivial duality between chiral (twisted chiral) superfields and linear (twisted linear) superfields.
} 
As $\Phi_{1}$ is semichiral $\left(\bar{D}_{+} \Phi_{1}=0\right), \Phi_{1}=\bar{D}_{+} \psi_{-}$for some unconstrained $\psi_{-}$, and varying $\psi_{-}$we get

$$
\bar{D}_{+}(\Lambda+\Phi)=0
$$

Similarly, we have

$$
\begin{aligned}
& \bar{D}_{-}(\Phi+\bar{\Lambda})=0, \\
& D_{+}(\bar{\Lambda}+\bar{\Phi})=0, \\
& D_{-}(\bar{\Phi}+\Lambda)=0 .
\end{aligned}
$$

From the above, we get

$$
\begin{aligned}
& \bar{D}_{+} D_{-}(\Phi-\bar{\Phi})=0, \\
& D_{+} \bar{D}_{-}(\Phi-\bar{\Phi})=0,
\end{aligned}
$$

which show that $\Phi$ is chiral superfield. Similarly $\Lambda$ is shown to be twisted chiral superfield, and we recover the superspace Lagrangian (60). Instead, if we vary $\theta, \phi, x, y$, we find the relations

$$
\begin{gathered}
e^{i \theta}=\left(\frac{\bar{\Phi}_{1}+\bar{\Phi}_{2}}{\Phi_{1}+\Phi_{2}}\right)^{\frac{1}{2}} \\
e^{i \phi}=\left(\frac{\bar{\Phi}_{1}+\Phi_{2}}{\Phi_{1}+\bar{\Phi}_{2}}\right)^{\frac{1}{2}} \\
-\frac{\ln (1+x)}{x}+2 y\left|\Phi_{1}+\bar{\Phi}_{2}\right|=0 \\
\frac{1}{y} \ln y+2\left|\Phi_{1}+\Phi_{2}\right|+2 x\left|\Phi_{1}+\bar{\Phi}_{2}\right|=0 .
\end{gathered}
$$

In this way we can express $\theta, \phi, x, y$ in terms of $\Phi_{1}, \bar{\Phi}_{1}, \Phi_{2}, \bar{\Phi}_{2}$ (at least implicitly).

Substituting back into (61) gives a new potential $K\left(\Phi_{1}, \bar{\Phi}_{1}, \Phi_{2}, \bar{\Phi}_{2}\right)$, and we can find the corresponding complex structures; in particular the anticommutator of these complex structures is proportional to the identity [9]. Our dual complex structures $K_{+}$and $K_{-}$are both hermitian and covariantly constant and their anticommutator has the same property as above.

$N=2$ superspace encodes complex structures: For example, chiral and twisted chiral superfields give complex structures $J_{ \pm}$with $\left[J_{+}, J_{-}\right]=0[\mathbb{8}]$. However, generic compact $W Z W$ models have $\left[J_{+}, J_{-}\right] \neq 0$. The orientation 
reversed complex structures $K_{ \pm}$on $S U(2) \times U(1)$ give an example since $\left[K_{+}, K_{-}\right] \neq 0$. We have found and presented an $N=2$ superspace description based on these complex structures, and hope that this may give insight into the general case.

\section{Acknowledgments}

It is a pleasure to thank Ulf Lindström for reading the manuscript and making many useful suggestions. We acknowledge partial support from the NFS under Grant No. PHY 9309888.

\section{References}

[1] E.S. Fradkin \& A.A. Tseytlin, Ann. Phys. 162 (1985) 31.

[2] T. Buscher, Phys. Lett. B159 (1985) 127, Phys. Lett. B194 (1987) 59, Phys. Lett. B201 (1988) 466.

[3] N. J. Hitchin, A. Karlhede, U. Lindström \& M. Roček, Commun. Math. Phys. 108 (1987) 535.

[4] M. Roček \& E. Verlinde, Nucl. Phys. B373 (1992) 630.

[5] A. Giveon, E. Rabinovici \& G. Veneziano, Nucl. Phys. B322 (1989) 167 ;

A. Shapere \& F. Wilzcek, Nucl. Phys. B320 (1989) 669;

A. Giveon, N. Malkin \& E. Rabinovici, Phys. Lett. B220 (1989) 551.

[6] S. Ferrara, D. Lüst, A. Shapere \& S. Theisen, Phys. Lett. B233 (1989) 147 ;

J. Lauer, J. Maas \& H.P. Nilles, Phys. Lett. B226 (1989) 251; Nucl. Phys. B351 (1991) 353;

W. Lerche, D. Lüst \& N.P. Warner, Phys. Lett. B231 (1989) 418;

S. Ferrara, D. Lüst \& S. Theisen, Phys. Lett. B233 (1989) 147;

A. Giveon, N. Malkin \& E. Rabinovici, Phys. Lett. B238 (1990) 57;

A. Font, L. Ibanez, D. Lust \& F. Quevedo, Phys. Lett. B245 (1990) 401 ; 
A. Giveon \& D.-J. Smit, Nucl. Phys. B349 (1991) 168;

A. Giveon \& M. Porrati, Phys. Lett. B246 (1990) 54; Nucl. Phys. B355 (1991) 422;

P. Candelas, X.C. de la Ossa, P.S. Green \& L. Parkes, Nucl. Phys. B359 (1991) 21;

T. Kugo \& B. Zwiebach, Prog. Theor. Phys. 87 (1992) 801.

[7] A. Giveon \& M. Roček, Nucl. Phys. B380 (1992) 128.

[8] S. J. Gates, C. M. Hull \& M. Roček, Nucl. Phys. B248 (1984) 157.

[9] T. Buscher, U. Lindström \& M. Roček., Phys. Lett. B202 (1988) 94.

[10] M. Roček, Modified Calabi-Yau Manifolds with Torsion, in 'Essays on Mirror Manifolds', ed. S. T. Yau (International Press, Hong Kong, 1992) 480.

[11] B. Zumino, Phys. Lett. B87 (1979) 203;

L. Alverez-Gaumé \& D. Z. Freedman, Commun. Math. Phys. 80 (1981) 443.

[12] B. B. Kim, SUNY at Stony Brook Ph.D. Thesis.

[13] M. Roček, K. J. Schoutens \& A. Sevrin., Phys. Lett. B265 (1991) 303. 